\title{
ROBUST BEAMFORMING VIA FIR FILTERING FOR GNSS MULTIPATH MITIGATION
}

\author{
Martí Mañosas-Caballú ${ }^{\dagger} \quad$ Gonzalo Seco-Granados ${ }^{\dagger} \quad$ A. Lee Swindlehurst ${ }^{\star}$ \\ ${ }^{\dagger}$ Dpt. Telecomm. and Syst. Engineering, Universitat Autònoma de Barcelona \\ ${ }^{\star}$ Dpt. Electrical Engineering and Comp. Science, University of California at Irvine
}

\begin{abstract}
This paper addresses the problem of multipath mitigation with GNSS antenna arrays. A beamformer that is able to cancel the multipath components regardless of their relative delay and directions of arrival is proposed. The weights are obtained from a set of spatial correlation matrices that allows us to estimate the multipath subspace. These matrices are generated after a FIR filter that reduces the correlation between the multipath components and the line-of-sight signal, and it is only used for spatial processing. Some representative simulation results show the multipath attenuation provided by the proposed method under different conditions.
\end{abstract}

Index Terms - Beamforming, GNSS, multipath, correlation.

\section{INTRODUCTION}

The term Global Navigation Satellite Systems (GNSS) is a generic expression referring to any system that enables the calculation of the user position based on signals transmitted by a constellation of satellites. Due to the operating principle of the GNSS, only the timedelay (referred to as code-phase) and the carrier-phase of the received Line-Of-Sight Signal (LOSS) bears useful information about the receiver position. Multipath reflections may bias the pseudoranges by several tens of meters, and at the same time, they hamper the ambiguity resolution process needed for carrier-phase ranging [1]. For this reason, significant research and development efforts have been devoted to the mitigation of multipath effects, and many techniques have been proposed so far [2]. However, these singleantenna techniques discriminate the LOSS from the reflections by using only temporal diversity, and hence, their performance is still insufficient for many precise applications.

In contrast, the use of multiple-antenna techniques in GNSS is a promising alternative. They exploit spatial diversity and hence they are able to discriminate the received signals when they come from different directions. The best and most well-known approaches are based on data-dependent beamforming, where the optimal beamforming weights depend on the statistics of the incoming data [3]. However, these methods fail in the presence of signals that are very correlated with the LOSS, and hence they are not useful to mitigate the multipath reflections with very small relative delay, i.e. coherent multipath. Some robust methods for highly correlated signals have been proposed, e.g. [4-12], but they present certain limitations.

In the field of GNSS, many beamforming techniques have been proposed so far that take into account the underlying particularities of a GNSS scenario, e.g. [13-31]. Since all present and planned navigation systems use a Direct-Sequence Spread-Spectrum (DSSS) signal, the information that is used to compute the weights can be extracted from the signal obtained either before or after the despreading process $[30,32]$. When mitigating the multipath is the main issue, the post-despreading signal is usually used since it contains the most noticeable contribution of the multipath. Although all robust GNSS methods improve on the performance of conventional beamforming techniques when coherent multipath is present, there are still important limitations.

In this work we address the problem of finding a beamformer that is robust against several GNSS multipath signals regardless of their relative delays and with arbitrary directions of arrival. In order to do so, we do not compute the weights from either the predespreading signal or the post-despreading signal. Instead, we propose to compute them from the output of an additional FIR filter that allows us to estimate the space spanned by the spatial signatures of the multipath reflections. This filter is only valid for the computation of the beamforming weights, which are then applied to either the pre-despreading signal or the post-despreading signal. The key idea behind the results presented in the paper comes from the model described in the next section. A description and justification of the proposed FIR method using this model is presented in Section 3, and some representative simulation results are found in Section 4.

\section{MODELING ASSUMPTIONS}

Let us consider that an arbitrary $m$-element array asynchronously receives the DS-SS signal transmitted by a given GNSS satellite together with $d<m$ multipath reflections. The $n$-th sample of the signal received by the array is modeled as:

$$
\mathbf{x}[n]=\sum_{k=0}^{d} \sum_{l=-\infty}^{\infty} \mathbf{a}_{k} \alpha_{k} b[l] c\left[n-\tau_{k}-l N\right]+\mathbf{u}[n]
$$

where $\mathbf{a}_{k} \in \mathbb{C}^{m}$ is the spatial signature of the $k$-th component, $\alpha_{k} \in$ $\mathbb{C}$ is its complex amplitude, and $\tau_{k} \in \mathbb{R}$ stands for its time-delay divided by the sampling period $T_{s} \in \mathbb{R}$. The index $k=0$ is reserved for the LOSS, so $\tau_{0}$ and the phase of $\alpha_{0}$ are the unknown code-phase and carrier-phase respectively, and $\mathbf{a}_{0}$ is assumed to be known up to a scaling factor. The sequence of symbols $b[l]$ forms the navigation message of the satellite, and it is assumed to be a stationary process. For its part, $c[n]:=c\left(n T_{s}\right)$ of length $N \in \mathbb{N}$ is the sampled version of the spreading code $c(t)$ of duration $T \in \mathbb{R}$, which is composed of a sequence of $P \in \mathbb{N}$ chips of duration $T_{c} \in \mathbb{R}$. Note that $\tau_{k}$ is not necessarily an integer number, but we use the notation $c\left[n-\tau_{k}\right]$ to denote the sampled version of $c\left(t-\tau_{k} T_{s}\right)$. Finally, $\mathbf{u}[n] \in \mathbb{C}^{m}$ contains the received noise at each element of the array, which is assumed to be spatially and temporarily white, and with identical noise power $\sigma_{u}^{2}$ at each sensor.

The signal obtained after despreading is a correlated version of the received signal (1) with a local discrete replica of the code $c(t)$, 
and it can be written as:

$$
\mathbf{y}[n]=\sum_{k=0}^{d} \sum_{l=-\infty}^{\infty} \mathbf{a}_{k} \alpha_{k} b[l] r_{c c}\left[n-\tau_{k}-l N\right]+\mathbf{r}_{\mathbf{u} c}[n]
$$

where:

$$
r_{c c}[n]:=\sum_{l=0}^{N-1} c[l+n] c[l] \quad \mathbf{r}_{\mathbf{u} c}[n]:=\sum_{l=0}^{N-1} \mathbf{u}[l+n] c[l]
$$

are the cross-correlation of $c[n]$ and $\mathbf{u}[n]$ with $c[n]$ respectively, and the output noise term, $\mathbf{r}_{\mathbf{u} c}[n]$, is no longer temporarily white, but only spatially white.

Generally, the post-despreading spatial correlation matrix $\mathbf{R}_{\mathbf{y y}}[n]=\mathrm{E}\left\{\mathbf{y}[n] \mathbf{y}[n]^{H}\right\}$ is used to calculate the beamforming weights, but only one estimate $\hat{\mathbf{R}}_{\mathbf{y y}}$ is calculated from all available samples. This is a problem since the information of the LOSS and multipath are mixed and cannot be distinguished properly. However, the output signal $\mathbf{y}[n]$ may be treated as a cyclostationary process, and hence $\mathbf{R}_{\mathbf{y y}}[n]$ cannot be considered constant but $N$-periodic. The MLE estimate of $\mathbf{R}_{\mathbf{y y}}[n]$ can be obtained as:

$$
\hat{\mathbf{R}}_{\mathbf{y y}}[n]=\frac{1}{L} \sum_{l=0}^{L-1} \mathbf{y}[n-l N] \mathbf{y}[n-l N]^{H}
$$

where $L$ out of $L N$ available samples are used.

In this work we calculate all possible correlation matrices in a period $N$ through (4). Then, their structure is exploited through the signal model presented in (2) to estimate the subspace spanned by the multipath spatial signatures $\mathbf{a}_{1}, \ldots, \mathbf{a}_{d}$ or multipath subspace. Once this is done, a beamformer that uses this subspace to mitigate the multipath is presented.

\section{GNSS MULTIPATH MITIGATION}

In this section we present and justify the proposed beamforming technique for multipath mitigation.

\subsection{Post-despreading Correlation Matrix}

For a given set of samples in a period $N$, let us say $n \in\{l N, l N+$ $1, \ldots, l N+N-1\}$ for some $l \in \mathbb{N}$, the output signal (2) can be approximately reduced to:

$$
\mathbf{y}[n] \approx \sum_{k=0}^{d} \mathbf{a}_{k} b[l] \phi_{k, n}+\mathbf{e}_{n}
$$

where $\tau_{k}<N \forall k$ is assumed for simplicity, $\phi_{k, n}:=\alpha_{k} r_{c c}[n-$ $\left.\tau_{k}-l N\right]$ and $\mathbf{e}_{n}:=\mathbf{r}_{\mathbf{u c}}[n]$. This approximation just neglects the contribution of $r_{c c}\left[n-\tau_{k}-l N\right]$ for those samples located far away from its peak. The notation $\phi_{k, n}$ is used to emphasize that the autocorrelation values are $r_{c c}\left[-\tau_{k}\right], r_{c c}\left[1-\tau_{k}\right], \ldots, r_{c c}\left[N-1-\tau_{k}\right]$ regardless of the value of $l$.

With this new formulation, $\mathbf{R}_{\mathbf{y y}}[n]$ can be written as:

$$
\mathbf{R}_{\mathbf{y y}}[n]=P_{b} \mathbf{A} \phi_{n} \boldsymbol{\phi}_{n}^{H} \mathbf{A}^{H}+\sigma_{e}^{2} \mathbf{I}
$$

where $P_{b}=\mathrm{E}\left\{b[l] b[l]^{*}\right\}, \phi_{n}=\left[\begin{array}{llll}\phi_{0, n} & \phi_{1, n} & \cdots & \phi_{d, n}\end{array}\right]^{T}, \mathbf{A}=$ $\left[\begin{array}{llll}\mathbf{a}_{0} & \mathbf{a}_{1} & \cdots & \mathbf{a}_{d}\end{array}\right], \mathbf{I}$ denotes the identity matrix, and $\sigma_{e}^{2}$ is the power of $\mathbf{e}_{n}$, which does not depend on $n$. As a result we have that $\mathbf{R}_{\mathbf{y} \mathbf{y}}[n]$ is the sum of a rank 1 positive definite signal matrix $\mathbf{A} \phi_{n} \boldsymbol{\phi}_{n}^{H} \mathbf{A}^{H}$

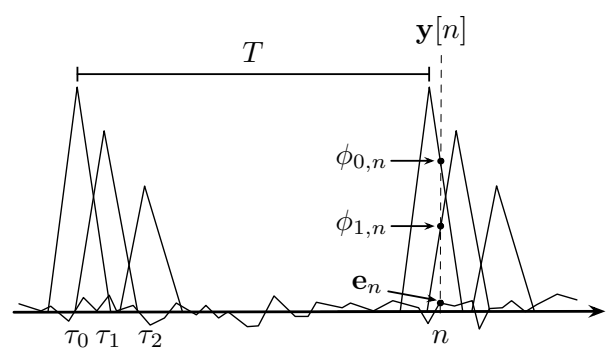

Fig. 1. Example of the underlying analog signal $\mathbf{y}(t)$ corresponding to $\mathbf{y}[n]$ as the sum of a signal term and a noise term, assuming $d=2$.

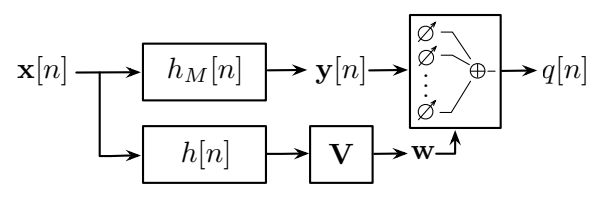

Fig. 2. Proposed processing with a new filter $h[n]$ parallel to the traditional matched filter $h_{M}[n]$ and followed by an estimation stage of $\mathbf{V}$, which finally produces the weights $\mathbf{w}$ to apply to $\mathbf{y}[n]$.

and a full-rank positive definite noise matrix $\sigma_{e}^{2} \mathbf{I}$. Thus, the eigendecompositon of $\mathbf{R}_{\mathbf{y y}}[n]$ has the particular property that its eigenvalues are $\lambda_{n, 1}>\lambda_{n, 2}=\ldots=\lambda_{n, m}=\sigma_{e}^{2}$ and the eigenvector associated with $\lambda_{n, 1}$ is a scaled version of $\mathbf{A} \phi_{n}$.

Now assume that we estimate $\mathbf{R}_{\mathbf{y y}}[n]$ for a given sample times $n_{1}, \ldots, n_{S}$ within a period $N$ through (4). Then the eigenvectors $\mathbf{v}_{1}, \ldots, \mathbf{v}_{S}$ corresponding to the maximum eigenvalues of $\mathbf{R}_{\mathbf{y y}}\left[n_{1}\right], \ldots, \mathbf{R}_{\mathbf{y y}}\left[n_{S}\right]$ can be obtained via an eigendecomposition. As they are a scaled version of the vectors $\mathbf{A} \phi_{n_{1}}, \ldots, \mathbf{A} \phi_{n_{S}}$, then the image space of the matrix $\mathbf{V}=\left[\mathbf{v}_{1} \cdots \mathbf{v}_{S}\right] \in \mathbb{C}^{m \times S}$ is:

$$
\operatorname{Im}\left[\mathbf{v}_{1} \cdots \mathbf{v}_{S}\right]=\operatorname{Im}\left[\mathbf{A} \phi_{n_{1}} \cdots \mathbf{A} \phi_{n_{S}}\right]=\operatorname{Im} \mathbf{A} \boldsymbol{\Phi}
$$

where $\boldsymbol{\Phi}=\left[\phi_{n_{1}} \cdots \phi_{n_{S}}\right]$ and Im denotes the image space. Finally, if the set $\left\{\phi_{n_{1}}, \ldots, \phi_{n_{S}}\right\}$ contains $d+1$ linearly independent vectors, then $\boldsymbol{\Phi}$ will be full row rank. As a consequence, $\operatorname{Im} \mathbf{V}$ will be $\operatorname{Im} \mathbf{A} \Phi=\operatorname{Im} \mathbf{A}$, which is the space spanned by all the spatial signatures. However, if the previous condition is not fulfilled, then $\operatorname{Im} \mathbf{A} \Phi \varsubsetneqq \operatorname{Im} \mathbf{A}$, and we do not obtain as much information as in the previous case.

As defined in (5), the coefficients $\phi_{k, n}$ are mainly the correlation contributions of the received signals after despreading. Hence, the shape of $r_{c c}[n]$ plays a decisive role in the linear independence among $\phi_{n_{1}}, \ldots, \phi_{n_{S}}$. Fig. 1 illustrates this observation and that samples close to $\tau_{0}$ will produce nearly linearly dependent vectors if the multipath signals are close enough to the LOSS, i.e. $\tau_{k}-\tau_{0}$ small enough for some $k \neq 0$, which results in a rank deficient matrix $\boldsymbol{\Phi}$. A natural question is then if there exists an approach different than despreading that provides a different $\boldsymbol{\Phi}$ with some structure that allows us to extract information about the multipath subspace from $\mathbf{V}$. As despreading can be modeled as a discrete matched filter with impulse response $h_{M}[n]=c^{*}[-n]$, using another FIR filter $h[n]$ seems a reasonable decision. As tracking cannot be properly achieved without $h_{M}[n]$, then $h[n]$ should be only used to calculate the multipath subspace and hence, the beamforming weights. Fig. 2 shows the proposed processing scheme in the case of applying $\mathbf{w}$ to $\mathbf{y}[n]$, which results in the signal $q[n]:=\mathbf{w}^{H} \mathbf{y}[n]$. 


\subsection{Inverse FIR Filtering}

As Fig. 1 shows, a filter $h[n]$ that gives a narrower response to $c[n]$ than $h_{M}[n]$ should greatly improve the structure of $\boldsymbol{\Phi}$. The narrowest possible response corresponds to:

$$
c[n] * h[n]=\delta[n]
$$

where $\delta[n]$ is the dirac impulse and $*$ denotes convolution. This obviously gives linearly independent vectors in $\boldsymbol{\Phi}$. However, the zeros in the spectrum of $c[n]$ produce a very high response in the spectrum of $h[n]$, which amplifies the input noise too much. Furthermore, a solution to (8) may not exist.

To avoid the first limitation, we introduce white noise $z[n]$ with power $\epsilon \in \mathbb{R}^{+}$together with $c[n]$ to the input of the filter to design. The idea behind this is that, in order for the output to be as close as possible to $\delta[n]$, the filter $h[n]$ should also mitigate the noise $z[n]$ since it is uncorrelated with $c[n]$. To address the second limitation, we find the filter that minimizes the error between the reference $\delta[n]$ and the real output as:

$$
\underset{\mathbf{h}}{\operatorname{argmin}} \mathrm{E}\left\{\|\mathbf{d}-(\mathbf{c}+\mathbf{z}) * \mathbf{h}\|^{2}\right\}
$$

where $\mathbf{d} \in \mathbb{R}^{2 N-1}, \mathbf{c} \in \mathbb{C}^{N}, \mathbf{z} \in \mathbb{C}^{N}$ and $\mathbf{h} \in \mathbb{C}^{N}$ are the column vectors that contain the samples of $\delta[n], c[n], z[n]$ and $h[n]$ respectively, and \|\| is the 2 -norm. Note that $h[n]$ is chosen to have the same length as the matched filter $h_{M}[n]$. After some manipulations, the solution to (9) becomes:

$$
\tilde{\mathbf{h}}=\left[\mathbf{R}_{\mathbf{c c}}+N \epsilon \mathbf{I}\right]^{-1} \mathbf{h}_{M} \quad \epsilon \in \mathbb{R}^{+}
$$

where $\mathbf{h}_{M} \in \mathbb{C}^{N}$ corresponds to $h_{M}[n]$ and $\mathbf{R}_{\text {cc }} \in \mathbb{C}^{N \times N}$ is defined as $\mathbf{R}_{\mathbf{c c}}(k, l)=r_{c c}[k-l]$.

The filter described by (10) has a degree of freedom given by $\epsilon$. This parameter allows us to vary the behaviour of the filter depending on the scenario of interest. However, note that $\tilde{\mathbf{h}}$ verifies $\lim _{\epsilon \rightarrow \infty} \tilde{\mathbf{h}} /\|\tilde{\mathbf{h}}\|=\mathbf{h}_{M} /\left\|\mathbf{h}_{M}\right\|$, and hence it only attains a scaled version of $\mathbf{h}_{M}$ for very large $\epsilon$. In order to overcome this limitation and obtain a more practical filter, we finally propose:

$$
\mathbf{h}=\left[(1-\rho) \mathbf{R}_{\mathbf{c c}}+\rho \mathbf{I}\right]^{-1} \mathbf{h}_{M} \quad \rho \in[0,1]
$$

which let the designer to obtain $\mathbf{h}_{M}$ and the same possible filters as in (10) up to a scaling factor using $\rho=N \epsilon /(1+N \epsilon)$. With this notation we have that $\rho=0$ corresponds to $\epsilon=0$, and hence we refer to the solution as the inverse filter or $\mathbf{h}_{I}$. In contrast, $\rho=1$ corresponds to $\epsilon \rightarrow \infty$, and we obtain $\mathbf{h}_{M}$. For $\rho \in(0,1)$ we obtain an intermediate behavior between $\mathbf{h}_{I}$ and $\mathbf{h}_{M}$. The lower $\rho$ is, the narrower the response to $c[n]$ is, but the lower the Signal-to-Noise Ratio (SNR) is, which gives us a trade-off between resolution and SNR. More precisely, the SNR at the output of the filter $\mathbf{h}$, which is defined as the ratio between the signal power and noise power at one of the peak samples, can be written as:

$$
\mathrm{SNR}=\mathrm{SNR}_{\mathrm{in}} \frac{\left(\mathbf{c}^{H}\left[(1-\rho) \mathbf{R}_{\mathbf{c c}}+\rho \mathbf{I}\right]^{-1} \mathbf{c}\right)^{2}}{\mathbf{c}^{H}\left[(1-\rho) \mathbf{R}_{\mathbf{c c}}+\rho \mathbf{I}\right]^{-2} \mathbf{c}}
$$

where $\mathrm{SNR}_{\text {in }}$ is the input SNR. Fig. 3 shows the SNR versus $\rho$ for different values of the sampling frequency $f_{s}$.

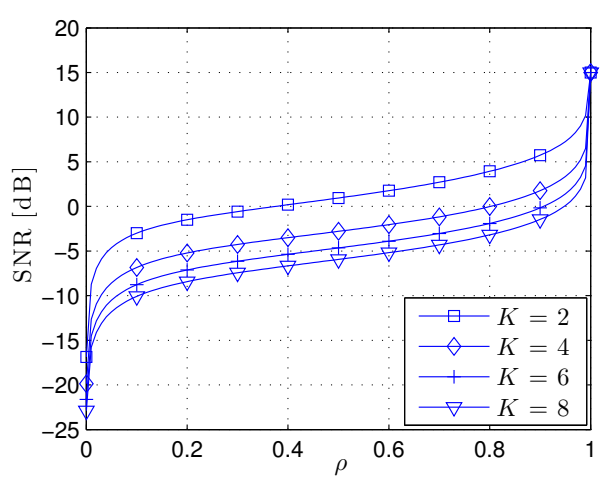

Fig. 3. SNR versus $\rho$ for $f_{s}$ equal to $K$ samples per chip. The $\mathrm{SNR}_{\text {in }}$ is computed assuming $\mathrm{CN}_{0}=45 \mathrm{~dB}-\mathrm{Hz}$ and the corresponding $f_{s}$.

\subsection{Multipath Subspace Estimation and Robust Beamforming}

The proposed method for estimating the multipath subspace starts by obtaining the matrix $\mathbf{V}$ from the output of the filter (11) only for those samples that contain signal contribution, since the other eigenvectors do not give information about the multipath subspace. Assuming that (8) holds, each signal contribution is only present in one sample and the maximum eigenvalue of $\mathbf{R}_{\mathbf{y y}}\left[n_{i}\right]$ is:

$$
\lambda_{i, 1}= \begin{cases}\sigma_{e}^{2} & \text { if } \nexists k: n_{i}=\tau_{k} \\ \sigma_{e}^{2}+m P_{k} \frac{\left(\mathbf{c}^{H} \mathbf{R c}\right)^{2}}{\|\mathbf{R c}\|^{2}} & \text { if } \exists k: n_{i}=\tau_{k}\end{cases}
$$

where $P_{k}:=\left|\alpha_{k}\right|^{2} P_{b}$ and $\mathbf{R}:=\left[(1-\rho) \mathbf{R}_{\mathbf{c c}}+\rho \mathbf{I}\right]^{-1}$.

From (13), a threshold $\lambda_{1}$ must be defined to determine whether a sample contains signal contribution or not. As $\mathbf{h}_{I}$ is an approximate solution of (8), each signal contribution may be present in more than one sample. Furthermore, in practice some estimation errors occur when calculating the eigenvalues. These two facts imply that the property (13) will hold only approximately, and hence $\lambda_{1}$ must not be as restrictive as $\sigma_{e}^{2}+m P_{k}\left(\mathbf{c}^{H} \mathbf{R c}\right)^{2} /\|\mathbf{R c}\|^{2}$, but instead, an intermediate value should be chosen between the two possible values presented in (13). As the values of $P_{k}$ are unknown, a reasonable choice is to always use a rough estimate of $P_{0}$ divided by some factor in order to also take the multipath into account. Those weak multipaths that do not exceed $\lambda_{1}$ will not be detected, but their impact can be neglected.

Once $\mathbf{V}$ is obtained, the LOSS contribution must be removed. Assuming that we use a sufficiently small sampling period (e.g. $T_{s} \leq \tau_{k}-\tau_{0} \forall k \neq 0$ when $\rho=0$ ), an eigenvector that only has a contribution from $\mathbf{a}_{0}$ exists, and the remaining ones only have multipath contributions. As a result, the entire LOSS contribution can be found by the following search:

$$
\underset{\mathbf{v}_{i}}{\operatorname{argmax}}\left|\mathbf{v}_{i}^{H} \mathbf{a}_{0}\right|
$$

Then, removing this vector from $\mathbf{V}$ results in a matrix $\overline{\mathbf{V}}$ that spans the multipath subspace. If the sampling period is not sufficiently small, equation (14) just allows us to remove a portion of the LOSS contribution, which worsens the estimation of the multipath subspace from $\overline{\mathbf{V}}$. However, the fact that $\overline{\mathbf{V}}$ contains a small contribution of $\mathbf{a}_{0}$ does not necessarily prevent the successful application of the method, as it will be shown in the numerical results. 
Once $\overline{\mathbf{V}}$ is estimated, designing the beamforming weights $\mathbf{w} \in$ $\mathbb{C}^{m}$ that mitigate the multipath is not an issue. We propose a distortionless beamformer, i.e. $\mathbf{w}^{H} \mathbf{a}_{0}=1$, that lies in the subspace orthogonal to $\operatorname{Im} \overline{\mathbf{V}}$, i.e. $\mathbf{w} \perp \operatorname{Im} \overline{\mathbf{V}}$. Among all possible solutions, a very straightforward one is:

$$
\mathbf{w}=\frac{\mathbf{P}_{\overline{\mathbf{V}}}^{\perp} \mathbf{a}_{0}}{\mathbf{a}_{0}^{H} \mathbf{P}_{\overline{\mathbf{V}}}^{\perp} \mathbf{a}_{0}}
$$

where $\mathbf{P}_{\overline{\mathbf{V}}}^{\perp}=\mathbf{I}-\overline{\mathbf{V}}\left(\overline{\mathbf{V}}^{H} \overline{\mathbf{V}}\right)^{-1} \overline{\mathbf{V}}^{H}$.

\subsection{Discussion}

The SNR at the output of our filter $\mathbf{h}$ is notably degraded for $\rho<1$, which renders the output signal useless for time-delay estimation by current single-antenna techniques. The impact of this low SNR behavior on the multipath estimation technique manifests itself in producing higher noise eigenvalues for $\mathbf{R}_{\mathbf{y y}}$. However, as $\hat{\mathbf{R}}_{\mathbf{y y}}$ is used in practice and the estimation errors increase if the SNR decreases, increasing the averaging time $L$ becomes indispensable. As a result, the lower the $\rho$, the higher the time devoted to multipath estimation must be, which yields a trade-off between resolution and computing time. A different way to improve resolution is to increase the sampling frequency $f_{s}:=1 / T_{s}$, since for $\rho=0$ the proposed method is able to mitigate those multipath reflections with $\tau_{k}-\tau_{0} \geq T_{s}$. But Fig. 3 shows that increasing $f_{s}$ gives lower SNR, so a similar trade-off is also present.

Finally, note that there exist some issues in a real system that have not been considered in our results and deserve special attention for future work. First, $\mathbf{h}$ will not be perfectly matched to the input, as the sampling of the input and reference signals are not necessarily the same. Second, the delay of each signal is not necessarily an integer number. As a result, when a signal or multipath is received, the corresponding output contribution may be distributed among two samples. This means that $\overline{\mathbf{V}}$ can still have a contribution from the LOSS after using (14), which worsens the performance of $\mathbf{w}$.

\section{NUMERICAL RESULTS}

This section presents some numerical examples of the proposed multipath mitigation technique in a scenario with $\mathrm{CN}_{0}=45 \mathrm{~dB}-\mathrm{Hz}$. We assumed that a Global Positioning System (GPS) LOSS signal and two multipaths are received by a 5-element antenna array with delays $\tau_{1}$ and $\tau_{2}$ so that $\tau_{1}-\tau_{0}=T_{c} / 4$ and $\tau_{2}-\tau_{0}=5 T_{c} / 4$. The GPS signal consists of several navigation data bits at a rate of $50 \mathrm{bps}$. Each bit contains 20 copies of a coarse acquisition (C/A) code of 1023 chips, so $T=1 \mathrm{~ms}$ and $T_{c} \approx 1 \mu \mathrm{s}$, and a rectangular pulse shaping is assumed for simplicity.

In order to contrast the resolution offered by the matched filter and the inverse filter, Figure 4 plots the corresponding noiseless outputs for a sampling frequency $f_{s}=8.184 \mathrm{MHz}$. It is very clear that $\mathbf{h}_{I}$ allows us to distinguish the 3 received signals. In contrast, $\mathbf{h}_{M}$ has such low resolution that the contribution of the 3 signals basically becomes one wide peak.

Figure 5 shows the performance obtained by the weights of (15) with $f_{s}=4.092 \mathrm{MHz}$. This value is chosen to prove that $T_{s}=\min \left\{\tau_{k}-\tau_{0}: k=1,2\right\}$ is small enough for the method to work satisfactorily. The figure plots the spatial attenuation $\left|\mathbf{w}^{H} \mathbf{a}_{0}\right|^{2} /\left(0.5\left|\mathbf{w}^{H} \mathbf{a}_{1}\right|^{2}+0.5\left|\mathbf{w}^{H} \mathbf{a}_{2}\right|^{2}\right)$ versus the value of $\rho$. For low $\rho$, the filter $\mathbf{h}$ gives very narrow peaks but the SNR is so low that the eigenvalue estimates are very noisy. Then, when $\rho$ is

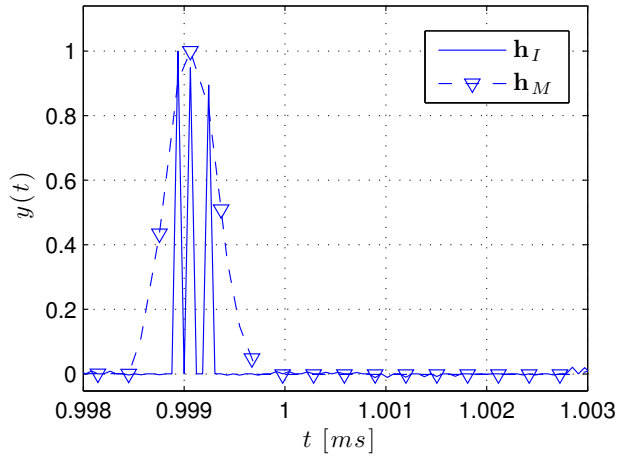

Fig. 4. Normalized noiseless output signal $y(t)$ from $\mathbf{h}_{M}$ and $\mathbf{h}_{I}$ versus time $t$. Scenario with sampling frequency $f_{s}=8.184 \mathrm{MHz}$, $\alpha_{1} / \alpha_{0}=0.9, \alpha_{2} / \alpha_{0}=0.8, \tau_{1}-\tau_{0}=T_{c} / 4$ and $\tau_{2}-\tau_{0}=5 T_{c} / 8$.

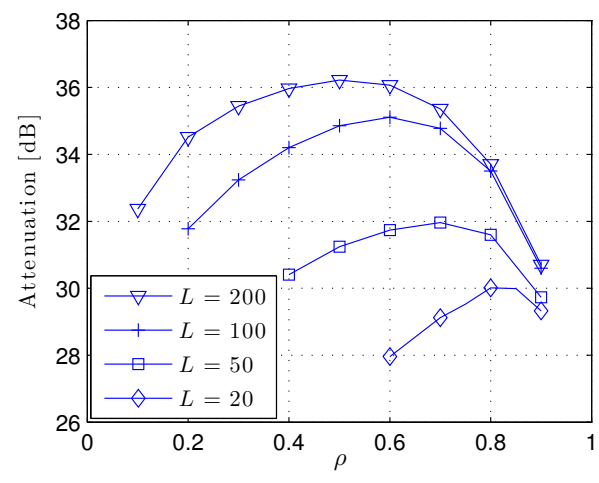

Fig. 5. Attenuation versus $\rho$. Scenario with sampling frequency $f_{s}=4.092 \mathrm{MHz}, \alpha_{1} / \alpha_{0}=0.9, \alpha_{2} / \alpha_{0}=0.8, \tau_{1}-\tau_{0}=T_{c} / 4$ and $\tau_{2}-\tau_{0}=5 T_{c} / 4$. The directions of arrival are $\theta_{0}=40^{\circ}, \theta_{1}=90^{\circ}$ and $\theta_{2}=140^{\circ}$.

increased, $\mathbf{h}$ gives wide peaks but the SNR increases and the performance improves. This happens until a certain point, where the peaks are so wide that $\overline{\mathbf{V}}$ has some contribution from $\mathbf{a}_{0}$. Then $\mathbf{w} \perp \overline{\mathbf{V}}$ also deletes the LOSS and the performance decreases. This explains why all curves show a maximum for $\rho \in(0,1)$. Finally note that when the averaging time $L$ increases, the low SNR consequences are mitigated and hence a lower $\rho$ can be used, so the position of the maximum is closer to $\rho=0$.

\section{CONCLUSION}

In this work we have proposed a novel technique to estimate the subspace spanned by the spatial signatures of the GNSS multipath signals received by an antenna array. In order to do so, a tunable FIR filter that offers a trade-off between resolution and SNR has been used. Then, a beamformer that lies on the subspace orthogonal to the estimated multipath subspace can be obtained. Our simulation results have shown that the proposed beamformer effectively attenuates the multipath signals for several values of the FIR filter design parameter, and that this parameter can be adjusted in order to obtain the balance between resolution and SNR that maximizes the multipath attenuation. 


\section{REFERENCES}

[1] B. Hofmann-Wellenhof, H. Lichtenegger, and J. Collins, Global Positioning System, Theory and Practice. SpringerVerlag Wien New York, 2001.

[2] M. Z. H. Bhuiyan and E. S. Lohan, "Advanced multipath mitigation techniques for satellite-based positioning applications," Int. Journal of Navig. and Obs., 2012.

[3] H. L. Van Trees, Optimum Array Processing (Detection, Estimation, and Modulation Theory, Part IV), 1st ed. WileyInterscience, Mar. 2002.

[4] B. Widrow, K. Duvall, R. Gooch, and W. Newman, "Signal cancellation phenomena in adaptive antennas: causes and cures," IEEE Trans. Antennas Propagat., vol. 30, no. 3, pp. 469-478, May 1982.

[5] M. D. Zoltowski and F. Haber, "A Vector Space Approach to Direction Finding in a Coherent Multipath Environment," IEEE Trans. on Antennas and Propag., vol. 34, no. 9, pp. 10691078, Sept. 1986.

[6] Y. Bresler, V. Reddy, and T. Kailath, "Optimum beamforming for coherent signal and interferences," IEEE Trans. ASSP, vol. 36, no. 6, pp. 833-843, Jun. 1988.

[7] S. Pillai and B. Kwon, "Forward/backward spatial smoothing techniques for coherent signal identification," IEEE Trans. ASSP, vol. 37, no. 1, pp. 8-15, Jan. 1989.

[8] I. Ziskind and M. Wax, "Maximum Likelihood Localization of Multiple Sources by Alternating Projection," IEEE Trans. ASSP, vol. ASSP-36, no. 10, pp. 1553-1560, Oct. 1988.

[9] F. Qian and B. D. V. Veen, "Quadratically constrained adaptive beamforming for coherent signals and interfence," Trans. on Signal Process., vol. 43, no. 8, pp. 1890-1900, 1995.

[10] T.-S. Lee and T.-T. Lin, "Reception of coherent signals with steering vector restoral beamformer," Signal Processing, vol. 72, no. 3, pp. 141-145, 1999.

[11] Y. Jiang, P. Stoica, Z. Wang, and J. Li, "Capon beamforming in the presence of steering vector errors and coherent signals," in Proc. ASAP Workshop, Lexington, MA, Jun. 2007.

[12] K. Ichige, H. Li, and H. Arai, "SURE: Simultaneous DOA and polarization estimation for arbitrary array configurations," in 7th IEEE Conf. on Sensor Array and Multichannel Signal Process., Yokohama, Japan, Jun. 2012, pp. 293-296.

[13] A. Naguib and A. Paulraj, "Performance of CDMA cellular networks with base-station antenna arrays," in Mobile Commun. Advan. Syst. and Components, ser. Lect. Notes in Comp. Science. Springer Berlin / Heidelberg, 1994, vol. 783, pp. 87-100.

[14] J. Miller and S. Miller, "An adaptive antenna array for multiple received signals in direct-sequence code-division multipleaccess communication systems," in Proc. IEEE MILCOM, Fort Monmouth, NJ, Oct. 1994, pp. 733-737.

[15] J. Ramos, M. Zoltowski, and M. Urgos, "Robust blind adaptive array. A prototype for GPS," in Proc. IEEE Int. Symp. on Phased Array Syst. and Tech., Boston, Massachusetts, Oct. 1996, pp. 406-410.

[16] D.-J. Moelker and Y. Bar-Ness, "An optimal array processor for GPS interference cancellation," in Proc. AIAA/IEEE Digital Avionics Syst. Conf., Atlanta, GA, Oct. 1996, pp. 285-291.
[17] G. Seco. and J. A. Fernández, "Multipath and Interference Errors Reduction in GNSS by Joint Pseudorange Measurement and Array Beamforming," in Proc. Europ. Sym. on GNSS, Munich, Germany, Apr. 1997, pp. 605-614.

[18] W. Myrick, M. Zoltowski, and J. Goldstein, "Anti-jam spacetime preprocessor for GPS based on multistage nested Wiener filter," in Proc. IEEE MILCOM, vol. 1, 1999, pp. 675-681.

[19] M. Amin, L. Zhao, and A. R. Lindsey, "Subspace array processing for the suppression of FM jamming in GPS receivers," IEEE Trans. on Aerospace and Electr. Syst., vol. 40, no. 1, pp. 80-92, Jan. 2000.

[20] R. L. Fante and J. J. Vaccaro, "Wideband cancellation of interference in a GPS receive array," IEEE Trans. on Aerospace and Electronic Syst., vol. 36, no. 2, pp. 549-564, Apr. 2000.

[21] R. G. Lorenz and S. P. Boyd, "Robust beamforming in GPS arrays," in Proc. ION Nation. Technic. Meeting, San Diego, CA, Jan. 2002.

[22] S.-J. Kim and R. A. Iltis, "GPS C/A code tracking with adaptive beamforming and jammering," in Proc. Asilomar Conf. on Signals, Syst. and Comput., vol. 2, Pacific Grove, CA, Nov. 2002, pp. 975-979.

[23] — - "STAP for GPS receiver synchronization," IEEE Trans. Aerosp. and Electr. Syst., vol. 40, no. 1, pp. 132-144, Jan. 2004.

[24] S. seung Hwang and J. J. Shynk, "An adaptive array based on composite and null despreaders for multiple GPS signals," in Proc. Asilomar Conf. on Signals, Syst. and Comput., Pacific Grove, CA, Nov. 2005, pp. 807-810.

[25] G. Seco-Granados, J. A. Fernandez-Rubio, and C. FernandezPrades, "ML estimator and hybrid beamformer for multipath and interference mitigation in GNSS receivers," IEEE Trans. on Signal Process., vol. 53, no. 3, pp. 1194-1208, Mar. 2005.

[26] M. Amin and W. Sun, "A novel interference suppression scheme for global navigation satellite systems using antenna array," IEEE Journal on Selected Areas in Commun., vol. 23, no. 5, pp. 999-1012, May 2005.

[27] W. Sun and M. Amin, "Maximum signal-to-noise ratio GPS anti-jam receiver with subspace tracking," in Proc. IEEE ICASSP, vol. 4, Philadelphia, PA, Mar. 2005, pp. 1085-1088.

[28] M. Sahmoudi and M. Amin, "Optimal robust beamforming for interference and multipath mitigation in GNSS arrays," in Proc. IEEE ICASSP, vol. 3, Honolulu, Hawaii, Apr. 2007.

[29] D. Lu, R. Wu, P. Li, and Z. Su, "GPS smart jammer suppressin algorithm based on spatial APES," in Proc. Int. Symp. on Intellig. Signal Process. and Commun. Syst., Xiamen, China, Dec. 2007, pp. 88-91.

[30] J. L. Vicario, F. Antreich, M. Barcelo, N. Basta, J. Cebrian, M. Cuntz, O. Gago, L. Gonzales, V. Heckler, C. Lavin, M. Manosas-Caballu, J. Picanyol, G. Seco-Granados, M. Sgammini, and F. Amarillo, "ADIBEAM: Adaptive digital beamforming for Galileo reference ground stations," in Proc. ION GNSS, Portland, OR, Sep. 2010, pp. 172-184.

[31] S. Backén, "On dynamic array processing for GNSS software receivers," Ph.D. dissertation, Lulea Univ. of Techn., 2011.

[32] D. de Lorenzo, F. Antreich, H. Denks, A. Hornbostel, C. Weber, and P. Enge, "Testing of adaptive beamsteering for interference rejection in GNSS receivers," in Proc. Europ. Navig. Conf. - GNSS, 2007. 